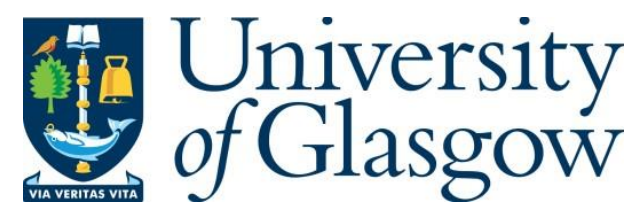

Murcia, P.R. (2019) Clinical insights: the equine microbiome. Equine Veterinary Journal, 51(6), pp. 714-715. (doi: 10.1111/evj.13165).

There may be differences between this version and the published version. You are advised to consult the publisher's version if you wish to cite from it.

This is the peer reviewed version of the following article:

Murcia, P.R. (2019) Clinical insights: the equine microbiome. Equine Veterinary Journal, 51(6), pp. 714-715, which has been published in final form at_ 10.1111/evj.13165. This article may be used for non-commercial purposes in accordance with Wiley Terms and Conditions for Self-Archiving.

http://eprints.gla.ac.uk/201055/

Deposited on: 21 November 2019

Enlighten - Research publications by members of the University of Glasgow http://eprints.gla.ac.uk 


\section{Clinical insights: The equine microbiome}

Horses, like any other animal (including humans) are hosts to a myriad of microorganisms that include bacteria, viruses, fungi and archaea, which collectively constitute the microbiome. Advances in sequencing technologies and bioinformatics over the last two decades have enabled researchers to draw an initial picture of the composition and structure of the microbiome in different species and in different body compartments. The advantage of highthroughput sequencing over previously used methods based on bacterial cultures or PCR approaches relies on the relatively unbiased nature of sequencing the bacterial 16S rRNA gene from biological specimens (1). While most microbiome studies have been carried out in humans and laboratory animals, the number of papers on microbiome research in domestic animals has increased significantly in recent years, and equine studies are not an exception. From those studies, it is now evident that the microbiome constitutes a complex and highly dynamic ecosystem and there is abundant evidence that changes in its composition are associated with health and disease. However, there is yet no consensus among researchers about how to define a healthy microbiome, as well as the mechanisms by which its structure impacts on disease outcomes.

The Equine Veterinary Journal (EVJ) has published a number of articles describing the equine microbiome that provided important insights on the range of microorganisms that cohabitate within horses under different physiological conditions. Most papers focused on the faecal microbiota (perhaps the most studied microbial community) as a proxy of the microorganisms inhabiting the gastrointestinal (GI) tract. As more than $50 \%$ of the total daily energetic requirements of the horse are the result of fermentation of dietary fibre, a process that takes place within the hindgut, it is obvious that the structure of the GI microbiome will play an important role not only in diseases of the GI but also on other important functions such as general metabolism and immunity. Given the relevance of the GI microbiome and the fact that animals originate from a sterile intrauterine environment, an important question is how the equine faecal microbiome develops from birth to adulthood. Two papers published in EVJ examined the colonisation dynamics of the gut microbiome. Costa et al. (2), performed a longitudinal study in which they characterised the faecal microbiome of mares and foals from day 1 to day 270 post-partum, divided in groups as follows: Day 1; Day 2-30, Day 31-60, Day 61-120; Day 121-180; Day 181-240, Day 240-270; adult mares. The authors generated and analysed a dataset with more than 4 million sequences that were classified in different phyla and genera and stratified by age. They observed significant changes in the abundance of phyla and genera among the different age groups. Moreover, when they visualized the microbiome community membership and structure, they observed that samples tended to cluster by age, consistent with dynamic changes in the microbiome that follow a time-dependent pattern. Specifically, this study showed that the foals' GI microbiome develops very rapidly (i.e. from day 1 post-partum) a rich and diverse intestinal microbiota that changes over time, with high abundance of transient organisms that over time are replaced by true colonisers. In addition, they showed that by 60 days post-partum, the structure of the faecal microbiome becomes more stable, and after 9 months it is more similar to that of their dams. In a more recent paper, Quercia et al. (3) applied a similar approach as Costa et al. (albeit with some methodological differences) to study the daily microbial colonisation of the gut from day 1 to day 10 post-partum. In this study, the authors examined thirteen mare-foal pairs, collecting for each pair not only faeces but also amniotic fluid, colostrum, milk, and foal meconium. This study design showed that the mare gut microbiota exhibited the highest level of biodiversity 
(measured as operational taxonomic unit [OTU] richness), followed by the meconium and then the amniotic fluid. Interestingly, gradual changes in the community structure of the microbiome were observed from meconium to day 3 post-partum, highlighting the dynamic nature of the microbiome as well as the power of the sequencing approach. While direct comparisons between the studies by Costa et al. and Quercia et al. are not straightforward, they both agree that from day 1 post-partum the gut microbiome shows a distinct community structure that varies over time and follows a convergent trajectory towards the mare gut microbiome, probably associated with early dietary changes, as foals start grazing and practice coprophagy during the first month of their lives.

Two papers published in EVJ examined the relationship between colic and changes in the microbiome. Weese et al. (4) performed a longitudinal case-control study that characterised the pre- and post-partum gut microbiome from mares kept in three different farms during the foaling season. To this end, they enrolled 221 mares and collected faecal samples at different timepoints before and after foaling date: approximately 14 days prior, within the first 4 days, and after 14-28 days. As 24 of the mares developed post-partum colic, they had a unique opportunity to compare the structure and composition of the gut microbiota before the animals develop colic. In addition, they also compared samples pre- and post-partum, as well as samples from pregnant and non-pregnant mares. Perhaps the most striking result from this study was the observation that faecal samples from mares with post-partum colic exhibited significantly higher diversity in their microbiome before the onset of colic, and such differences were more pronounced in samples that preceded a colic episode by less than 10 days, suggesting a potential risk association between changes in microbiome and colic. In particular, they observed a lower relative abundance of Firmicutes and Bacteroidetes and an increase in the relative abundance of Proteobacteria. In addition, Weese et al. also detected differences in the gut microbiome associated with late pregnancy. With regard to the observed microbiome changes in samples before the onset of colic, the authors cautiously noted that causation was not proved but differences were suggestive of a role in the microbiota in the development of colic.

More recently, Stewart et al. (5) published in EVJ a prospective observational clinical study that compared the faecal microbiome in horses presenting to a tertiary referral hospital for colic $(n=17)$ vs. an elective surgical procedure $(N=30)$. Consistent with other studies, the authors observed significant differences in the gut microbiome between the two groups. Specifically, the intestinal microbiota of horses with colic displayed fewer species and lower diversity. This was in line with the observations made by Weese et al., reinforcing the general concept that a reduction in bacterial richness and diversity is associated with gastrointestinal disease.

While all the aforementioned studies explored changes in the gut microbiome under different physiological (pregnancy/non-pregnancy, pre- and post-partum, and age [particularly early times after birth]) and pathological conditions (colic vs non-colic), two separate papers by Schoster et al., examined the effect of external factors. The first paper described an experimental trial involving eight healthy horses in which the impact of transport, fasting and anaesthesia on the faecal microbiome was determined (6). The motivation for this study was the general perception that horses subject to general anaesthesia and surgery occasionally develop GI disease, and this could be associated with changes in the microbiome linked not only to anaesthesia and surgery, but also to transport related stress and/or fasting that precedes surgical procedures. Briefly, horses were transported to a research facility where they were fasted for 12 hours before anaesthesia. After six hours of anaesthesia, horses were 
allowed to recover, and food was available six hours later. Faecal samples were obtained at various time-points: the day before transportation, after 12 hours of fasting, and on day 1, 2 and 3 after anaesthesia. Significant changes in the number of phyla, classes, orders and families were detected, and a lower abundance of Clostridiales was observed. However, some parameters such as alpha diversity (i.e. the number of OTUs) were unaltered, overall suggesting that transport, fasting and anaesthesia might have some effect on the intestinal microbiota of healthy horses.

Another paper by Schoster et al., this time a randomised placebo-controlled field trial, studied the longitudinal effect of a multi-strain probiotic containing lactobacilli and Bifidobacterium (7). This study enrolled 38 neonatal foals at three days of age, which were given the probiotic $(n=21)$ or placebo $(n=17)$ daily for 3 weeks. Faecal swabs were collected from each foal every 2 weeks and standard microbiome analyses were performed. Surprisingly, very limited changes (compared to the control group) were detected in the microbiome of the animals that received the probiotic, suggesting a limited effect of the treatment. Of note, the authors raised caution on the interpretation of their results as further studies using higher doses of probiotics should be performed in order to reach more robust conclusions.

We are still in the early days of microbiome research and while the number of published studies on the subject are significantly increasing, we have not yet reached the point in which the patterns of microbiome diversity in health and disease among different species have been systematically described. This gap in our knowledge translates into questions of clinical importance. For example, while changes in microbiome have been observed in health and disease (e.g. colic), these studies do not prove causation. Further, microbiome sequencing studies like the ones described here have several limitations: current sequencing approaches lack resolution at lower taxonomic levels (i.e. species level); the presence of bacterial DNA does not necessarily mean the presence of living bacteria and does not provide an explicit characterisation of their function. Logistical constrains limit the number of animals available, and field studies, while essential to understand natural conditions, are subject to multiple biases such as differences in animal management between farms. From a clinical perspective, future studies should lead to the identification of microbiome markers associated with health and disease that would ideally allow the detection of certain diseases before clinical onset, or treatment evaluation. From a therapeutic perspective, research could lead to treatments based on the transplant of healthy microbiome to combat infections by pathogenic bacteria, thus reducing antibiotic usage and the emergence of antibiotic resistance.

\section{P. R. Murcia}

MRC-University of Glasgow Centre for Virus Research

Glasgow, Scotland, United Kingdom

E-mail: Pablo.Murcia@Glasgow.ac.uk

\section{References}

1. L. W. Hugerth, A. F. Andersson, Analysing Microbial Community Composition through Amplicon Sequencing: From Sampling to Hypothesis Testing. Front Microbiol 8, 1561 (2017).

2. M. C. Costa, H. R. Stampfli, E. Allen-Vercoe, J. S. Weese, Development of the faecal microbiota in foals. Equine Vet J 48, 681-688 (2016). 
3. S. Quercia et al., Early colonisation and temporal dynamics of the gut microbial ecosystem in Standardbred foals. Equine Vet J 51, 231-237 (2019).

4. J. S. Weese et al., Changes in the faecal microbiota of mares precede the development of post partum colic. Equine Vet J 47, 641-649 (2015).

5. H. L. Stewart et al., Differences in the equine faecal microbiota between horses presenting to a tertiary referral hospital for colic compared with an elective surgical procedure. Equine Vet J 51, 336-342 (2019).

6. A. Schoster, M. Mosing, M. Jalali, H. R. Staempfli, J. S. Weese, Effects of transport, fasting and anaesthesia on the faecal microbiota of healthy adult horses. Equine Vet J 48, 595-602 (2016).

7. A. Schoster et al., The longitudinal effect of a multi-strain probiotic on the intestinal bacterial microbiota of neonatal foals. Equine Vet J 48, 689-696 (2016). 